\title{
Damage Characterization Method for Structural Health Management Using Reduced Number of Sensor Inputs
}

\author{
T. Krishnamurthy ${ }^{*}$, Jacob D. Hochhalter \\ NASA Langley Research Center, Hampton, VA 23681, U.S.A. \\ and \\ Adam M. Gallegos \\ Lockheed Martin Information Systems and Global Solutions, Hampton, VA 23681, U.S.A.
}

\begin{abstract}
A major concern in aircraft structures is the growth of undetected damage (i.e. cracks) due to fatigue and low velocity foreign object impact that can reach a critical size during flight, resulting in loss of control of the aircraft. In this paper, a damage detection method using strain measurements at selected sensor locations is proposed. An optimization procedure combined with the Finite Element Method (FEM) was developed to detect damage in structures. It is demonstrated that the strains measured at a limited number of sensor locations can be effectively used to predict the location, size, and orientation of the damage. The procedure described can be used to characterize the damage in a Structural Health Management (SHM) system once fully developed. The damage is characterized by size, location, and orientation angle. Numerical examples are presented to demonstrate an optimization procedure in several damage configurations. It is found that the damage size and the location were estimated for a constant orientation angle within five percent for all the configurations analyzed. However, the gradient based optimization procedure fails if the orientation angle is also included in the estimation. Conversely, it is demonstrated that a Genetic Algorithm (GA) estimates all the damage parameters, including the orientation angle, to within five percent. However the GA requires a large number of finite element analyses to estimate the damage parameters and hence is computationally expensive.
\end{abstract}

\section{Introduction}

The development of validated multidisciplinary Integrated Vehicle Health Management (IVHM) technologies to prevent loss of control of aircraft due to adverse conditions remains a safety-related challenge facing the next generation of aircraft. The adverse conditions include environmental factors, actuator and sensor faults or failures, and damage conditions. A major concern in aircraft structures is the growth of undetected damage (i.e. cracks) due to fatigue and low velocity foreign object impact that can reach a critical size during flight, resulting in loss of control of the aircraft. Hence, development of efficient methodologies to determine the presence, location, and severity of damage in critical structural components is highly important in developing efficient Structural Health Management (SHM) systems.

Approaches for the detection of damage size and location in structures can be based on changes in vibration or ultrasonic wave characteristics caused by the damage [1, 2, 3]. Approaches that use vibration characteristics are effective for detecting relatively large damage, since smaller damage sizes may have only negligible effects on vibration properties. The approach utilizing ultrasonic wave characteristics is effective in detecting smaller damage, but generally requires a dense network of sensors. Even with the continuous advancement in these approaches, there still are large uncertainties associated with the determination of damage size, location, and orientation. Hence, there is a need to increase the level of confidence in detecting the damage size, location, and orientation. Recently an attempt was made to predict damage size and location using the EXtended Finite Element Method (X-FEM) and the measured displacement field from the sensors [4]. The method in [4] fails to predict the orientation angle of damage due to the current limitation in the X-FEM implementation in the analysis software used. Since strains are directly measured, additional computational efforts are needed to convert the strains measured at sensor locations to displacements [5]. Hence, a damage detection method using the sensor strains rather than the displacements is highly desirable.

\footnotetext{
* Aerospace Engineer, Durability and Damage Tolerance, Associate Fellow, AIAA
} 
Fiber Optics Strain Sensing (FOSS) technology offers the ability to obtain strain measurements with low weight addition to the structures [6]. Therefore, strain fields are used herein to characterize the damage. The objective of this paper is to extend the work performed in [4] to achieve the following:

1. Use strains from sensors to detect the damage rather than displacements.

2. Reduce the number of sensor measurements needed to detect the damage location, size and orientation angle.

3. Use conventional Finite Element Method (FEM) rather than X-FEM.

In this paper, a damage characterization method using a finite element method for Structural Health Management (SHM) is proposed. A study of two different optimization procedures (a gradient-based and a non-gradient-based method) is presented to estimate the damage size, location, and orientation. Next, several numerical examples are presented to demonstrate the effectiveness of the method. Finally, a brief summary is presented.

\section{Development of the Objective Function for the Optimization Procedure}

For the present study, damage in a two-dimensional geometry is considered. The damage is assumed to be in the form of a crack in a plate with height $h$ and width $w$ as shown in figure 1 . The damage is characterized by four independent variables as shown in the Figure 1. The first two variables are the location of the center of the damage, $X_{r}$ and $Y_{r}$, the third variable is the damage size, $a_{r}$, and the fourth variable is the damage orientation angle, $\theta_{\mathrm{r}}$, measured with respect to the $\mathrm{X}$-axis. These four independent variables, defining the damage, are referred to as the damage configuration in this paper.

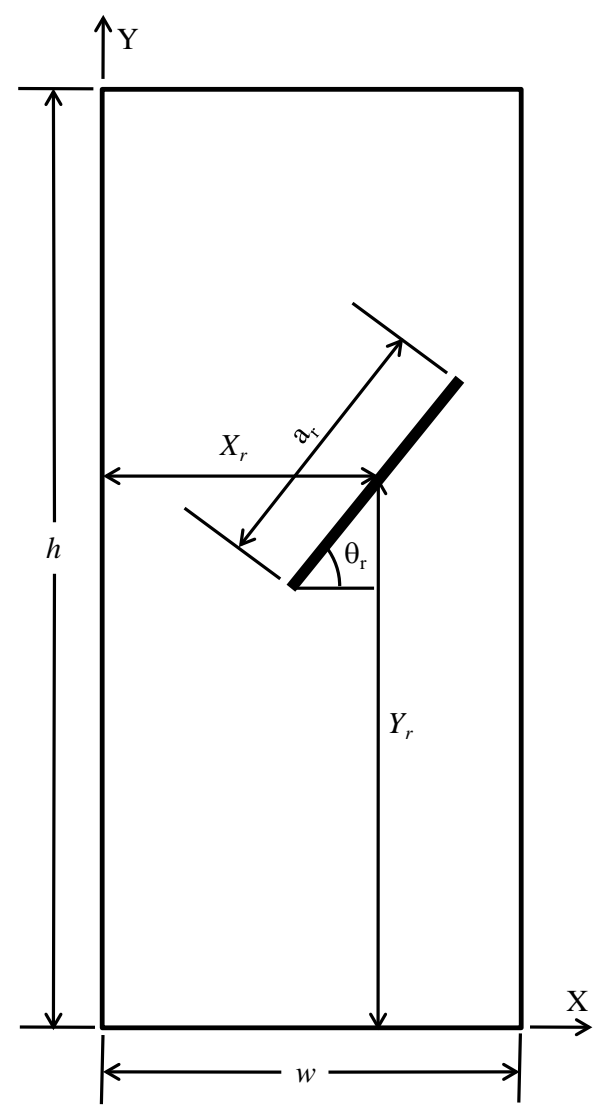

Figure 1. Schematic of plate geometry and the four variables used to define the damage configuration. 
Consider an arbitrary reference damage in a plate as shown in Figure 2 with damage location $\mathrm{X}_{\mathrm{r}}$ and $\mathrm{Y}_{\mathrm{r}}$, damage size, $\mathrm{a}_{\mathrm{r}}$, and damage orientation angle, $\theta_{\mathrm{r}}$. It is assumed that strains from pre-selected sensor locations are known for this reference damage configuration. The sensor locations are shown schematically in Figure 2 with filled circles.

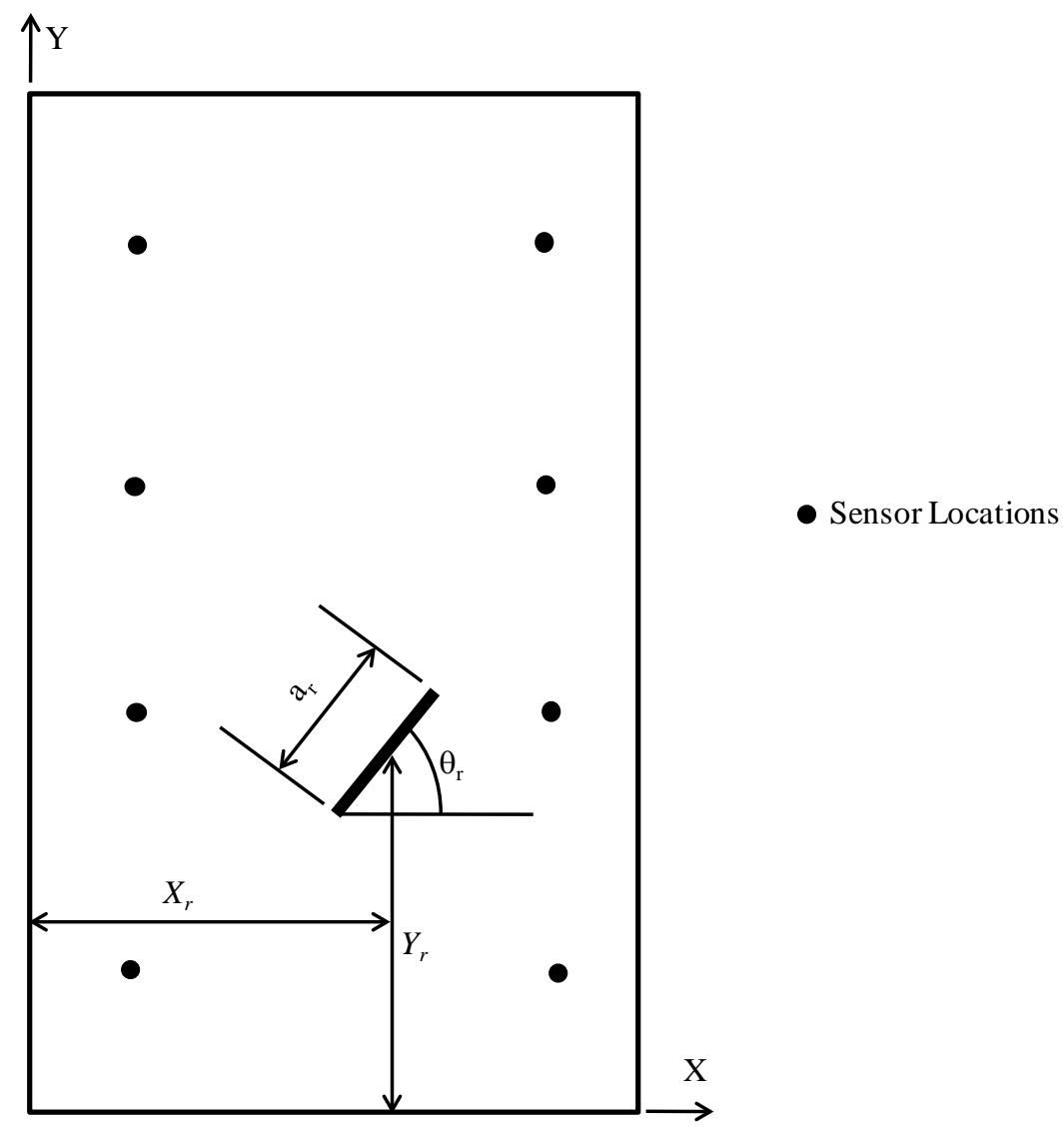

Figure 2. Schematic of damage configuration and sensor locations.

In the optimization procedure, the damage location, $X_{r}$ and $Y_{r}$, damage size, $a_{r}$, and damage orientation angle, $\theta_{\mathrm{r}}$, are treated as unknowns. The three Cartesian strains $\left(\varepsilon_{X}^{r}, \varepsilon_{Y}^{r}, \gamma_{X Y}^{r}\right)$ measured at sensor locations due to the damage are called the reference solution in this paper. The reference strain, $\left\{S^{r}\right\}_{j}$, at any sensor location $j$ can be denoted as

$$
\left\{S^{r}\right\}_{j}=\left\{\begin{array}{c}
\varepsilon_{X}^{r} \\
\varepsilon_{Y}^{r} \\
\gamma_{X Y}^{r}
\end{array}\right\}_{j}
$$

The reference strains at the sensor locations along with their coordinates are used in computing the objective function.

The process of estimating the damage configuration begins by building a finite element model with an initial guess for the damage configuration, as shown in Figure 3. The finite element mesh is not shown in Figure 3 for clarity. An optimization procedure must then be used to iterate the damage configuration to minimize the objective function. A finite element analysis is performed at each iteration, and the strains at the sensor locations are computed. 


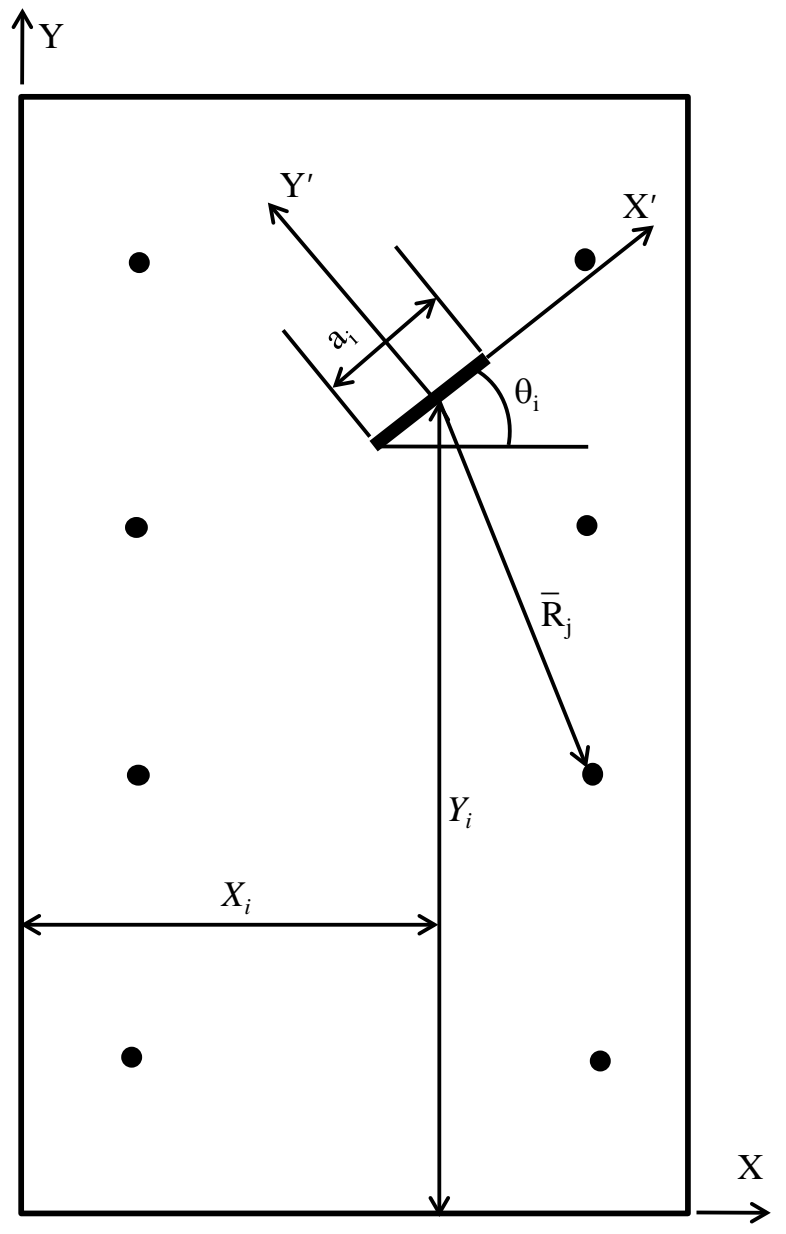

Figure 3. Finite element model damage configuration for the $i^{\text {th }}$ iteration.

Let the strains at the $j^{\text {th }}$ sensor location from the finite element solution at the $i^{\text {th }}$ iteration be denoted as

$$
\left\{S^{i}\right\}_{j}=\left\{\begin{array}{c}
\varepsilon_{X}^{i} \\
\varepsilon_{Y}^{i} \\
\gamma_{X Y}^{i}
\end{array}\right\}_{j}
$$

If the damage configuration for the current iteration matches the reference damage configuration, the strains in Equations (1.1) and (1.2) will be identical at all sensor locations. If the reference sensor strains in Equation (1.1) are not the same as the finite element strains in Equation (1.2) at all the sensor locations, another finite element model is built with a new assumed arbitrary damage configuration. In order to automate and reduce the number of iterations needed to match the strains from the reference solution and the arbitrary configuration, an optimization procedure is utilized:

1. Start the optimization procedure with an arbitrary damage location, size and orientation angle. Construct a finite element model for the current assumed damage configuration.

2. Perform a finite element analysis for the current damage configuration and compute strains at all the $N$ sensor locations.

3. Find the sum of the root square error between the reference strain in Equation (1.1) and the computed finite element strain in Equation (1.2) as 


$$
\phi=\left[\sum_{j=1}^{N}\left(\left\{S^{r}\right\}_{j}-\left\{S^{i}\right\}_{j}\right)\left(\left\{S^{r}\right\}_{j}-\left\{S^{i}\right\}_{j}\right)^{T}\right]^{\frac{1}{2}}
$$

Where $T$ in Equation (1.3) denotes the transpose of the vector. The $\phi$ in the Equation (1.3) is generally referred as an objective or error function in the optimization procedure.

4. When the estimated value for the objective function $\phi$ in Equation (1.3) is less than a pre-selected tolerance (ideally it should be exactly zero), convergence is achieved and the iteration is stopped. Then, the current damage configuration is the estimated damage configuration.

5. If convergence is not achieved, the optimization algorithm will estimate a new damage configuration and the Steps 2-4 are repeated until convergence is achieved.

It is found that better convergence is achieved in the optimization procedure if the strains in Equations (1.1) and (1.2) are transformed to a new $X^{\prime}-Y^{\prime}$ axis rotated by the orientation angle $\theta_{i}$ for the current damage configuration. The rotated $X^{\prime}-Y^{\prime}$ is shown in Figure 3. The transformed strains in Equations (1.1) and (1.2) are obtained from

$$
\begin{aligned}
& \left\{S^{r}\right\}_{j}=\left(\begin{array}{ccc}
\cos ^{2} \theta_{i} & \sin ^{2} \theta_{i} & \sin \theta_{i} \cos \theta_{i} \\
\sin ^{2} \theta_{i} & \cos ^{2} \theta_{i} & -\sin \theta_{i} \cos \theta_{i} \\
2 \sin \theta_{i} \cos \theta_{i} & -2 \sin \theta_{i} \cos \theta_{i} & \left(\cos ^{2} \theta_{i}-\sin ^{2} \theta_{i}\right)
\end{array}\right)\left\{\begin{array}{c}
\varepsilon_{X}^{r} \\
\varepsilon_{Y}^{r} \\
\gamma_{X Y}^{r}
\end{array}\right\}_{j} \\
& \left\{S^{i}\right\}_{j}=\left(\begin{array}{ccc}
\cos ^{2} \theta_{i} & \sin ^{2} \theta_{i} & \sin \theta_{i} \cos \theta_{i} \\
\sin ^{2} \theta_{i} & \cos ^{2} \theta_{i} & -\sin \theta_{i} \cos \theta_{i} \\
2 \sin \theta_{i} \cos \theta_{i} & -2 \sin \theta_{i} \cos \theta_{i} & \left(\cos ^{2} \theta_{i}-\sin \theta^{2}\right)
\end{array}\right)\left\{\begin{array}{c}
\varepsilon_{X}^{i} \\
\varepsilon_{Y}^{i} \\
\gamma_{X Y}^{i}
\end{array}\right\}_{j}
\end{aligned}
$$

Also, it is found that much better convergence is achieved in optimization procedure if the strains in Equations (1.4) and (1.5) are multiplied by a weight factor equal to the radial distance $\bar{R}_{j}$ from the center of the current damage configuration to the sensor location at $j$ (shown schematically in Figure 3). The weight factor $\bar{R}_{j}$ ensures that weighted importance is given for the sensor strains measured far away from the damage. The modified objective function in Equation (1.3) is as follows

$$
\phi=\left[\sum_{j=1}^{N}\left(\left\{S^{r}\right\}_{j}-\left\{S^{i}\right\}_{j}\right)\left(\left\{S^{r}\right\}_{j}-\left\{S^{i}\right\}_{j}\right)^{T} \bar{R}_{j}^{2}\right]^{\frac{1}{2}}
$$

The estimation of the damage configuration using the optimization procedure is demonstrated through numerical examples in subsequent sections. The following numerical examples are presented:

1. where locations $X_{r}$ and $Y_{r}$ are unknown;

2. where locations $X_{r}, Y_{r}$ and the damage size $a_{r}$ are unknown;

3. where all four damage configuration variables $\left(X_{r}, Y_{r}, a_{r}\right.$ and $\left.\theta_{r}\right)$ are unknown.

The accuracy and reliability of estimating the damage configuration are largely dependent on the number of sensor locations at which strains are available for the structure. In general, the larger the number of sensors, the greater the accuracy in detecting the damage configuration.

\section{Numerical Examples}

The damage estimation is demonstrated on damage fully contained within a two-dimensional plate. The plate geometry, boundary conditions and material properties along with the finite element model used in the analyses for a reference damage configuration is shown on the deformed plate geometry in Figure 4. It is necessary for each iteration in the optimization procedure that a new finite element model with the current damage configuration be created. 


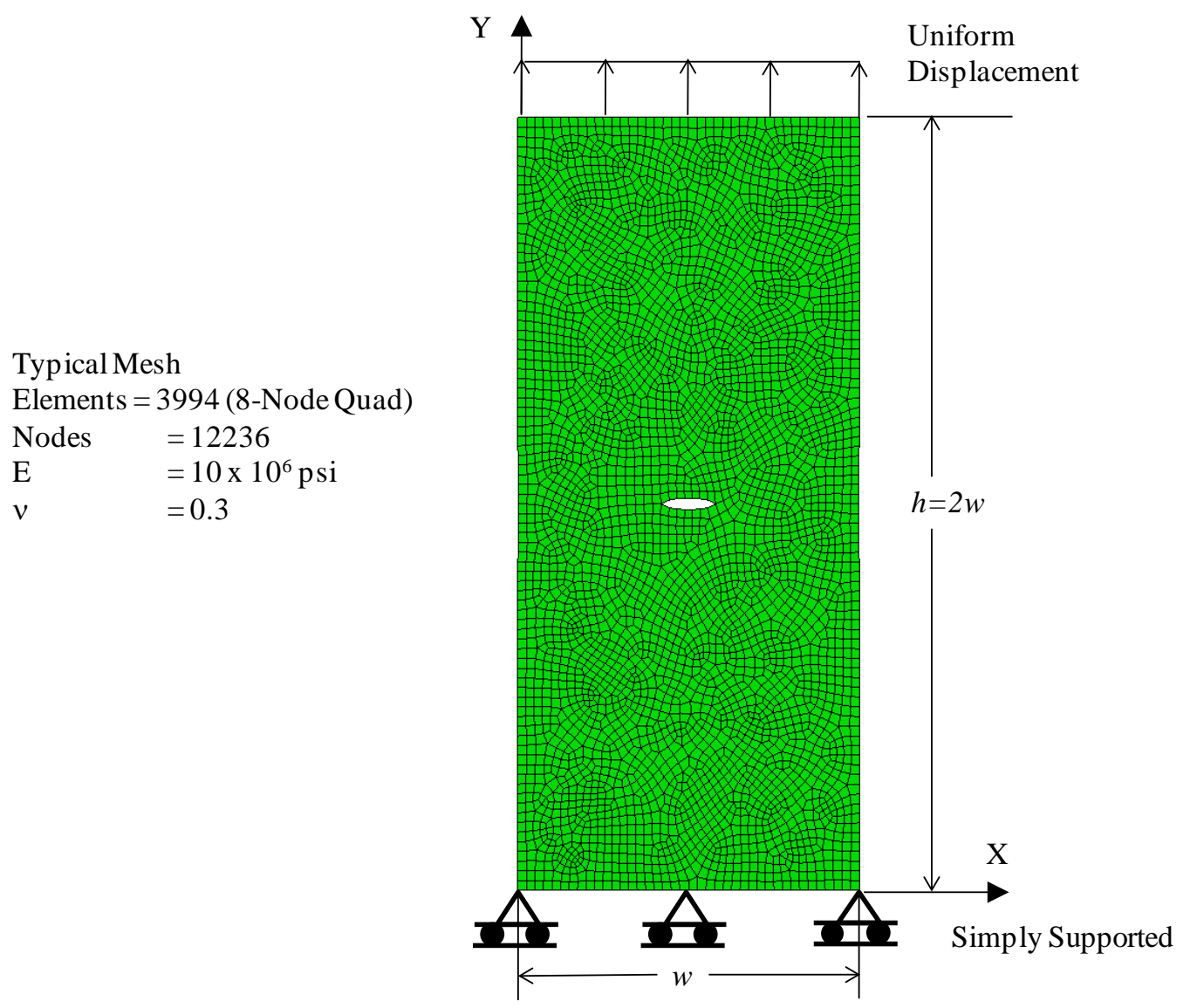

Figure 4. Finite element model for an example damage configuration.

The mesh is created by defining three mesh size constraints: at the crack tip, at the center of the crack, and at the outer boundary of the plate. The mesh then conforms to those size constraints within the domain. To determine the typical mesh size constraints required, the objective function, Equation (1.6), was computed with $S^{i}=0$. The mesh size constraints are refined until this computed value was not dependent on small perturbations of the mesh. Since it is intractable to complete a similar convergence study for each finite element analysis preformed during the optimization procedure, those mesh size constraints that provided a converged value were used in subsequent finite element analyses.

\section{Reference solution generation:}

The strains at the sensor locations are the only input to the optimization procedure discussed in Section II apart from loading and boundary conditions. For this paper, synthetic strain data from pre-selected sensor locations are generated by performing a finite element analysis of the reference damage configuration. The strains at the sensor locations are interpolated from the element strains in the finite element analysis. This synthetic strain data is then used as the reference solution, which is estimated by the optimization procedure.

Two reference damage configurations were considered for the numerical examples presented in this paper and are shown on the deformed plate geometry in Figure 5. Case I is a center damage configuration, while Case II is an upper right damage configuration.

For Case I, the center damage configuration, the reference location is $X_{r}=0.5 \mathrm{~W}, \mathrm{Y}_{\mathrm{r}}=0.5 \mathrm{~h}$, and the damage size is $\mathrm{a}_{\mathrm{r}}$ $=0.167 \mathrm{w}$. Reference damage orientations are $\theta_{\mathrm{r}}=0,15$, and $50 \mathrm{deg}$.

For Case II, the upper right damage configuration, the reference location is $\mathrm{X}_{\mathrm{r}}=0.667 \mathrm{w}, \mathrm{Y}_{\mathrm{r}}=0.75 \mathrm{~h}$, and the damage size is $\mathrm{a}_{\mathrm{r}}=0.167 \mathrm{w}$. Reference damage orientations are $\theta_{\mathrm{r}}=0,15$, and 50 deg. 


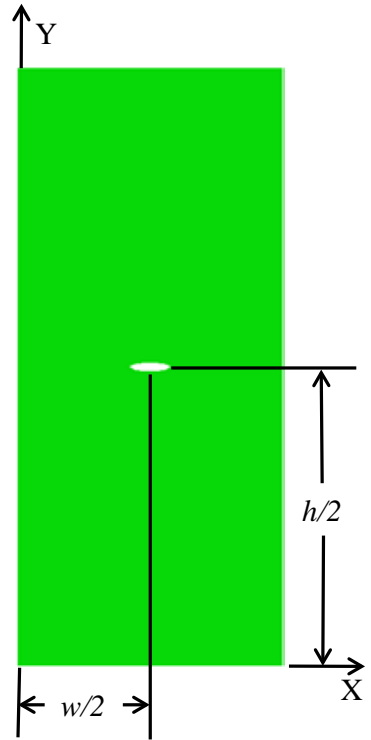

Case I: Center Crack Configuration

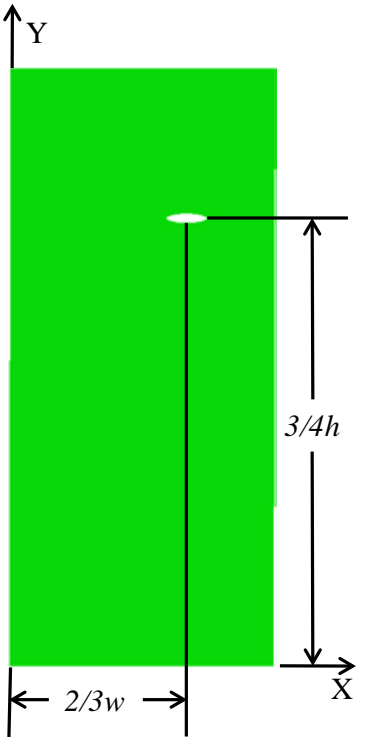

Case II: Upper Right Crack Configuration

Figure 5: Two reference damage configurations considered in the examples, showing $\theta_{\mathrm{r}}=0 \mathrm{deg}$.

\section{Investigating the use of a reduced sensor density}

The number of sensor locations needed to detect a damage configuration with a given accuracy depends upon the damage location, size, and orientation angle. In the method presented in [4], it was assumed that the reference data were known for the entire plate geometry. This is not a practical assumption. From the numerical examples presented in this paper, it is concluded that four columns of seven sensors for a total of twenty-eight sensors were sufficient to detect the damage configurations to within five percent error. However, twenty-eight sensors may not represent the minimum possible density of sensors to ensure accurate damage detection in the numerical examples presented. It represents, however, a practical sensor density. The twenty-eight sensors are arranged as shown in Figure 6. The twenty-eight sensor locations were used for all the damage configurations in Case I and Case II mentioned in the previous section.

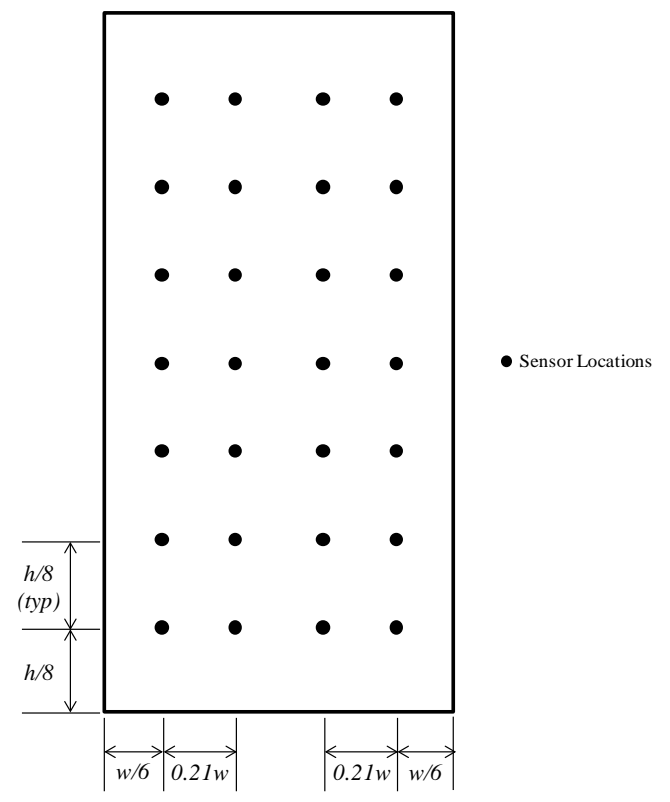

Figure 6. Twenty-eight sensor locations used in the example problems. 'typ' denotes that the dimension is repeated in that direction. 


\section{III.a. Gradient-Based Optimization Study}

To minimize the objective function defined in Equation (1.6) an optimization procedure must be used. In this section, the capability of a gradient-based technique is investigated to minimize the objection function, i.e. to estimate the damage configuration. DOT uses an unconstrained gradient-based technique to minimize the objective function and was used in this study [7].

\section{Damage Location as the Unknown Variable}

Next, the optimization procedure is demonstrated in the two-unknown variable example. The damage location, $\mathrm{X}_{\mathrm{r}}$ and $Y_{r}$, were treated as the two unknowns in the problem. For this example, the reference Case I and Case II damage configurations shown in Figure 5 were used.

Case I center damage configuration: The optimization procedure described in Section II was started with an initial guess of the damage location set to $X_{i}=0.25 \mathrm{w}$ and $Y_{i}=0.167 \mathrm{~h}$. The final location of the damage was determined from the optimization procedure. The results from the optimization procedure are presented in Table 3 . It can be seen from Table 3, both $X_{r}$ and $Y_{r}$ locations of the damage are detected to within 0.5 percent.

Table 3. Case I center damage configuration: Two-unknown variables

\begin{tabular}{|c|cc|cc|cc|cc|}
\hline & \multicolumn{2}{|c|}{ Reference Configuration } & \multicolumn{2}{|c|}{ Initial Guess } & \multicolumn{2}{|c|}{ Optimization } & \multicolumn{2}{c|}{ \% Difference from } \\
Case & $\mathrm{Y}_{\mathrm{r}}$ & $\mathrm{X}_{\mathrm{r}}$ & $\mathrm{Y}_{\mathrm{i}}$ & $\mathrm{X}_{\mathrm{i}}$ & $\mathrm{Y}_{\mathrm{f}}$ & $\mathrm{X}_{\mathrm{f}}$ & $\mathrm{Y}_{\text {error }}$ & $\mathrm{X}_{\text {error }}$ \\
\hline $\mathrm{I}$ & $0.500 \mathrm{~h}$ & $0.500 \mathrm{w}$ & $0.167 \mathrm{~h}$ & $0.250 \mathrm{w}$ & $0.499 \mathrm{~h}$ & $0.502 \mathrm{w}$ & 0.23 & 0.31 \\
\hline
\end{tabular}

Case II upper right damage configuration: The optimization procedure described in Section II was started with an initial guess of the damage location set to $X_{i}=0.25 \mathrm{w}$ and $Y_{i}=0.167 \mathrm{~h}$. The final location of the damage is determined from the optimization procedure. The results from the optimization procedure are presented in Table 4. For the Case II damage configuration, the damage locations, $X_{r}$ and $Y_{r}$, are estimated to within one percent.

Table 4. Case II upper right damage configuration: Two-unknown variable

\begin{tabular}{|c|c|c|c|c|c|c|c|c|}
\hline \multirow[b]{2}{*}{ Case } & \multicolumn{2}{|c|}{ Reference Configuration } & \multicolumn{2}{|c|}{ Initial Guess } & \multicolumn{2}{|c|}{$\begin{array}{l}\text { Optimization } \\
\text { Result }\end{array}$} & \multicolumn{2}{|c|}{$\begin{array}{l}\text { \% Difference from } \\
\text { Reference Configuration }\end{array}$} \\
\hline & $\mathrm{Y}_{\mathrm{r}}$ & $X_{r}$ & $\mathrm{Y}_{\mathrm{i}}$ & $X_{i}$ & $Y_{f}$ & $X_{f}$ & $\mathrm{Y}_{\text {error }}$ & $\mathrm{X}_{\text {error }}$ \\
\hline II & $0.750 \mathrm{~h}$ & $0.667 \mathrm{w}$ & $0.167 \mathrm{~h}$ & $0.250 \mathrm{w}$ & $0.744 \mathrm{~h}$ & $0.660 \mathrm{w}$ & 0.93 & 0.78 \\
\hline
\end{tabular}

The numerical examples presented the two-unknown variables problem demonstrated that the optimization procedure developed in this paper estimates the damage location to within one percent.

\section{Damage Location and Size as the Unknown Variables}

For this three-unknown variables problem, the locations, $X_{r}$ and $Y_{r}$, and the damage size, $a_{r}$, are treated as unknowns in the optimization procedure keeping the orientation angle constant. The orientation angle is fixed at zero degrees. Here, both the Case I and Case II reference configurations were considered. The results from these studies are described in this section.

Case I center damage configuration: The optimization procedure described in Section II is started with an initial guess of the damage location and size set to $X_{i}=0.25 \mathrm{w}, Y_{i}=0.167 \mathrm{~h}$, and $a_{i}=0.083 \mathrm{w}$. The final location and size of the damage are determined from the optimization procedure. The results from the optimization procedure are presented in Table 5. 
Table 5. Case I center damage configuration: Three-unknown variables

\begin{tabular}{|c|c|c|c|c|c|c|c|c|c|c|c|c|}
\hline \multirow[b]{2}{*}{ Case } & \multicolumn{3}{|c|}{ Reference Configuration } & \multicolumn{3}{|c|}{ Initial Guess } & \multicolumn{3}{|c|}{ Optimization Result } & \multicolumn{3}{|c|}{$\begin{array}{c}\text { \% Difference from } \\
\text { Reference Configuration }\end{array}$} \\
\hline & $\mathrm{Y}_{\mathrm{r}}$ & $a_{r}$ & $X_{r}$ & $Y_{i}$ & $a_{i}$ & $X_{i}$ & $Y_{f}$ & $a_{f}$ & $X_{f}$ & $\mathrm{Y}_{\text {error }}$ & $\mathrm{a}_{\text {error }}$ & $X_{\text {error }}$ \\
\hline I & $0.500 \mathrm{~h}$ & $167 \mathrm{w}$ & $500 \mathrm{w}$ & .167l & $.083 \mathrm{w}$ & 0.250 & $0.499 \mathrm{~h}$ & 0.16 & v & 0.25 & 3.78 & 1.73 \\
\hline
\end{tabular}

For the three-unknown variables example, the damage size, $\mathrm{a}_{\mathrm{r}}$, is predicted to within four percent and the damage location, $\mathrm{X}_{\mathrm{r}}$ and $\mathrm{Y}_{\mathrm{r}}$, are predicted to within two percent.

Case II upper right damage configuration: The optimization procedure described in Section II is started with an initial guess of the damage location and size set to $X_{i}=0.25 \mathrm{w}, Y_{i}=0.167 \mathrm{~h}$, and $\mathrm{a}_{\mathrm{i}}=0.083 \mathrm{w}$. The results from the optimization procedure are presented in Table 6.

Table 6. Case II upper right damage configuration: Three-unknown variables

\begin{tabular}{|c|ccc|ccc|ccc|ccc|}
\hline & \multicolumn{3}{|c|}{ Reference Configuration } & \multicolumn{3}{|c|}{ Initial Guess } & \multicolumn{3}{c|}{ Optimization Result } & \multicolumn{3}{c|}{ \% Difference from } \\
Reference Configuration \\
Case & $\mathrm{Y}_{\mathrm{r}}$ & $\mathrm{a}_{\mathrm{r}}$ & $\mathrm{X}_{\mathrm{r}}$ & $\mathrm{Y}_{\mathrm{i}}$ & $\mathrm{a}_{\mathrm{i}}$ & $\mathrm{X}_{\mathrm{i}}$ & $\mathrm{Y}_{\mathrm{f}}$ & $\mathrm{a}_{\mathrm{f}}$ & $\mathrm{X}_{\mathrm{f}}$ & $\mathrm{Y}_{\text {error }}$ & $\mathrm{a}_{\text {error }}$ & $\mathrm{X}_{\text {error }}$ \\
\hline II & $0.750 \mathrm{~h}$ & $0.167 \mathrm{w}$ & $0.667 \mathrm{w}$ & $0.167 \mathrm{~h}$ & $0.083 \mathrm{w}$ & $0.250 \mathrm{w}$ & $0.743 \mathrm{~h}$ & $0.163 \mathrm{w}$ & $0.658 \mathrm{w}$ & 0.92 & 2.44 & 1.27 \\
\hline
\end{tabular}

For this Case II damage configuration, the damage size, $\mathrm{a}_{\mathrm{r}}$, is predicted to within three percent, and the damage location, $\mathrm{X}_{\mathrm{r}}$ and $\mathrm{Y}_{\mathrm{r}}$, are predicted to within two percent.

The numerical examples presented so far with the zero degree orientation angle demonstrated that the damage size and the location can be estimated accurately using the optimization procedure.

\section{Damage Location, Size, and Orientation Angle as the Unknown Variables}

For the four-unknown variables example, the damage location, $X_{r}$ and $Y_{r}$, the damage size, $a_{r}$, and the orientation angle, $\theta_{\mathrm{r}}$, are unknown. The reference damage configurations in Figure 5 were used with two orientation angles, $\theta_{\mathrm{r}}=$ $50 \mathrm{deg}$. and $\theta_{\mathrm{r}}=15 \mathrm{deg}$. The reference center damage configurations for two orientation angles are shown on the deformed plate geometry in Figure 9. Similarly, the reference upper right damage configurations for two orientations angle are shown on the deformed plate geometry in Figure 10.

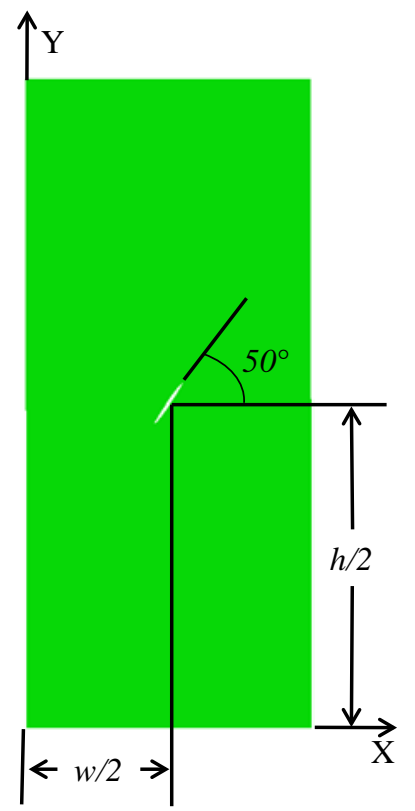

Case I-C1: $50^{\circ}$ Center Damage Configuration

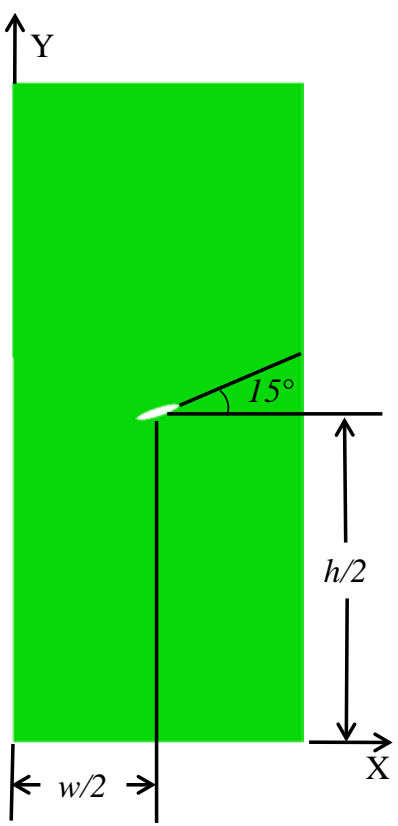

Case I-C2: $15^{\circ}$ Center Damage Configuration

Figure 9. Reference center damage configurations with non zero orientation angles. 

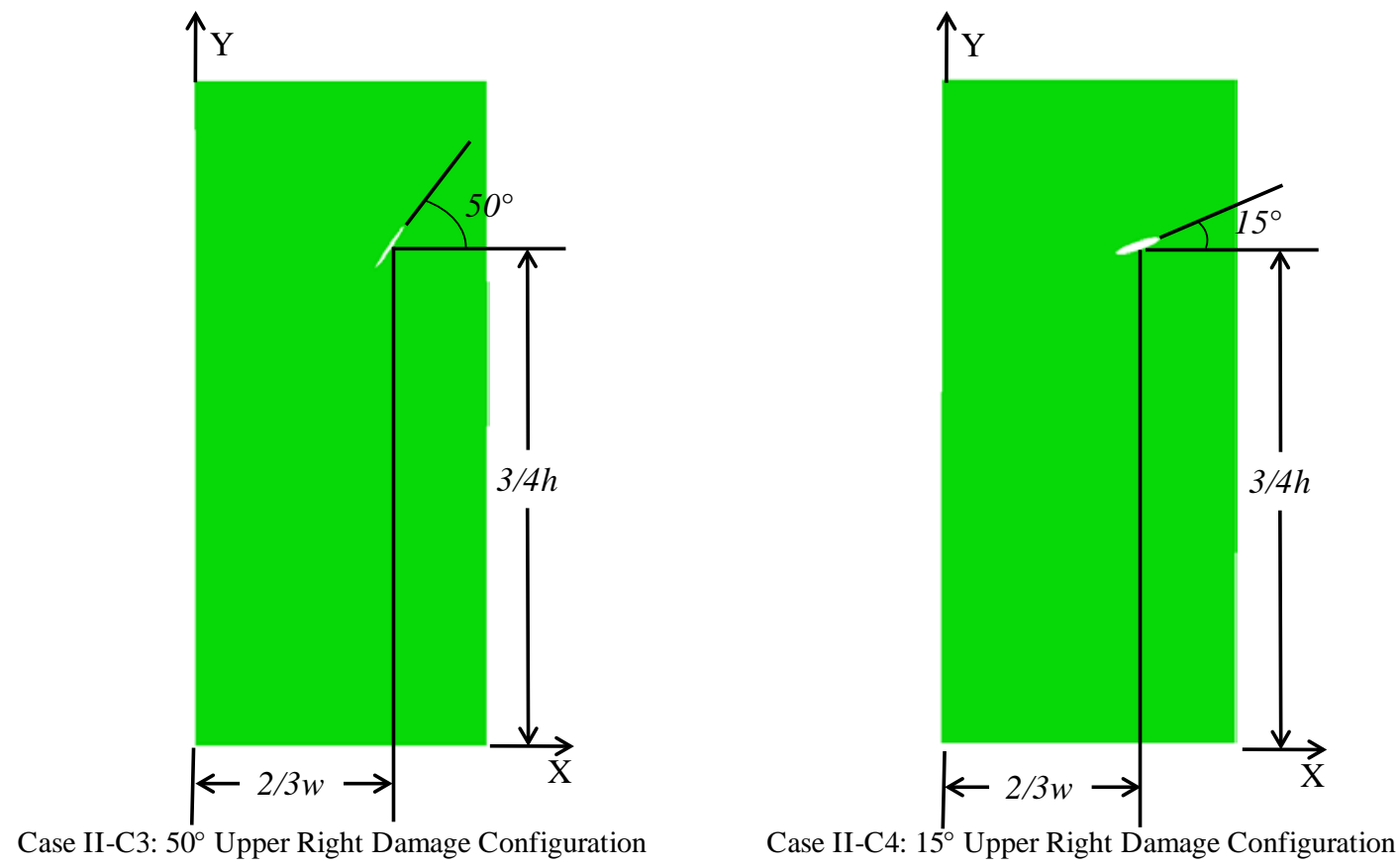

Figure 10. Reference upper right damage configurations with non zero orientation angles.

The gradient-based optimization procedure for the four variables problem failed to converge to the correct damage configuration. The solution converges to a local minimum rather than the absolute minimum of the objective function. Hence it was decided to use non-gradient-based optimization algorithm to obtain the global minimum solution rather than a local minimum.

\section{III.b. Non-Gradient-Based Genetic Algorithm}

The genetic algorithm (GA) implemented in Matlab [8], was investigated since it is a method for solving highly nonlinear objective functions and is globally convergent, as opposed to the gradient-based technique, which was shown to converge at local minima. Unlike the gradient-based technique, which generates a single trial point at each iteration, the GA generates a population of points at each iteration. The GA then selects those trial points from the population that are the most fit, i.e. most closely matches the target solution, and generates a new population of points based on the fittest members. For a complete discussion of the Matlab GA implementation, see [8].

Some potential downsides of the GA are:

- Since the trial points in the first iteration are randomly generated, there is no guarantee that the optimization will produce equivalent converged solutions upon repeated trials;

- There are several input options that must be provided and those that provide the fastest, most consistent convergence must be determined by trial-and-error and;

- More function calls are required (in this case, finite element analyses).

It was found that the following GA-input options provided consistent convergence behavior for the problem under investigation:

- Population size: 20

- Creation function: Feasible population

- Selection: Tournament

- Mutation: Adaptive feasible

- Crossover: Two point 
This population size was large enough to provide sufficient variation diversity, while minimizing the number of function calls required. By using the 'Feasible population' Creation function and 'Adaptive feasible' Mutation, the trial points were guaranteed to stay contained within the upper and lower bounds on the damage configuration. This was important because damage configurations that lie outside that domain would potentially create damage that was not fully contained within the plate, which were considered invalid possibilities in this study. Tournament Selection chooses parents to the next generation by randomly sampling the current population and keeping only the fittest. One crucial behavior that these inputs provide is the ability to maintain diversity in the population after several iterations. The GA algorithm is applied to solve the reference configurations shown in Figure 9 and Figure 10.

The results from GA algorithm for the two reference center damage configurations (Figure 9) are shown in Table 7. Similarly, the GA algorithm results for the two reference upper right damage configurations (Figure 10) are shown in Table 8.

Table 7. Case I center damage configurations: Four-unknown variables

\begin{tabular}{|l|cccc|cccc|ccccc|}
\hline & \multicolumn{3}{|c|}{ Reference Configuration } & \multicolumn{4}{c|}{ Optimization Result } & \multicolumn{4}{c|}{ \% Difference from } \\
Ceference Configuration \\
Case & $\mathrm{Y}_{\mathrm{r}}$ & $\mathrm{a}_{\mathrm{r}}$ & $\mathrm{X}_{\mathrm{r}}$ & $\theta_{\mathrm{r}}$ & $\mathrm{Y}_{\mathrm{f}}$ & $\mathrm{a}_{\mathrm{f}}$ & $\mathrm{X}_{\mathrm{f}}$ & $\theta_{\mathrm{f}}$ & $\mathrm{Y}_{\text {error }} \mathrm{a}_{\text {error }}$ & $\mathrm{X}_{\text {error }}$ & $\theta_{\text {error }}$ \\
\hline $\mathrm{I}-\mathrm{C} 1$ & $0.500 \mathrm{~h}$ & $0.167 \mathrm{w}$ & $0.500 \mathrm{w}$ & $50^{\circ}$ & $0.500 \mathrm{~h}$ & $0.166 \mathrm{w}$ & $0.501 \mathrm{w}$ & $49.8^{\circ}$ & 0.07 & 0.40 & 0.13 & 0.50 \\
\hline $\mathrm{I}-\mathrm{C} 2$ & $0.500 \mathrm{~h}$ & $0.167 \mathrm{w}$ & $0.500 \mathrm{w}$ & $15^{\circ}$ & $0.500 \mathrm{~h}$ & $0.167 \mathrm{w}$ & $0.501 \mathrm{w}$ & $15.2^{\circ}$ & 0.07 & 0.2 & 0.13 & 1.39 \\
\hline
\end{tabular}

Table 8. Case II upper right damage configurations: Four unknown variables

\begin{tabular}{|l|cccc|cccc|cccc|}
\hline & \multicolumn{3}{|c|}{ Reference Configuration } & \multicolumn{3}{c|}{ Optimization Result } & \multicolumn{4}{c|}{ \% Difference from } \\
Case enence Configuration \\
& $\mathrm{Y}_{\mathrm{r}}$ & $\mathrm{a}_{\mathrm{r}}$ & $\mathrm{X}_{\mathrm{r}}$ & $\theta_{\mathrm{r}}$ & $\mathrm{Y}_{\mathrm{f}}$ & $\mathrm{a}_{\mathrm{f}}$ & $\mathrm{X}_{\mathrm{f}}$ & $\theta_{\mathrm{f}}$ & $\mathrm{Y}_{\text {error }}$ & $\mathrm{a}_{\text {error }}$ & $\mathrm{X}_{\text {error }}$ & $\theta_{\text {error }}$ \\
\hline II-C3 & $0.750 \mathrm{~h}$ & $0.167 \mathrm{w}$ & $0.667 \mathrm{w}$ & $50^{\circ}$ & $0.752 \mathrm{~h}$ & $0.172 \mathrm{w}$ & $0.668 \mathrm{w}$ & $51.8^{\circ}$ & 0.22 & 3.40 & 0.12 & 3.71 \\
\hline II-C4 & $0.750 \mathrm{~h}$ & $0.167 \mathrm{w}$ & $0.667 \mathrm{w}$ & $15^{\circ}$ & $0.751 \mathrm{~h}$ & $0.167 \mathrm{w}$ & $0.667 \mathrm{w}$ & $15.2^{\circ}$ & 0.06 & 0.2 & 0.00 & 1.27 \\
\hline
\end{tabular}

From the Tables 7 and 8, it is seen that the GA resulted in more accurate damage detection than the gradient-based method because of its global convergence characteristic. However, the improved accuracy comes at the cost of running more than $\mathrm{O}(2000)$ finite element analyses, as opposed to $\mathrm{O}(100)$ with the gradient-based techniques. More research is needed to reduce the computational cost in the GA for the class of examples attempted in this paper.

\section{Summary}

In this paper, a damage characterization method using strain measured at selected sensor locations is proposed. An optimization procedure combined with the finite element method was developed to predict damage. It is demonstrated that the strains measured at a limited number of sensor locations can be effectively used to predict the location and size of the damage.

The damage is characterized by size, location, and orientation angle. Numerical examples were presented to demonstrate the gradient-based optimization procedure in several damage configurations. First, the damage location was estimated keeping the damage size and orientation angle constant. It was found that the damage location was estimated to within one percent for all the damage configurations tested. Next, both the damage size and the location were estimated keeping the orientation angle constant. It was found that the size and location were estimated to within five percent for all the configurations analyzed. However the gradient-based optimization procedure failed if the orientation angle was also included in the estimation.

It is demonstrated that a Genetic Algorithm (GA) estimates all the damage parameters including the orientation angle to within five percent. However, the GA requires a large number of finite element analyses to estimate the damage parameters and hence is computationally expensive. None the less, the numerical examples show that using a GA along with finite element analysis provides a methodology to determine the damage configuration in structural health management systems. 


\section{References}

1. Kim, J.T. and Stubbs, N., "Improved Damage Identification Method Based on Modal Information," Journal of Sound and Vibration, Vol. 252, pp. 223-238, 2002.

2. Mal, A.K., Ricci, F., Banerjee, S., and Shih, F., "A Conceptual Structural Health Monitoring System Based on Vibration and Wave Propagation," Structural Health Monitoring: An International Journal, Vol. 4, pp. 283 - 293, 2005.

3. Wang, L., and Yuan, F. G.,"Active Damage Localization Technique Based on Energy Propagation of Lamb Waves," Smart Structures and Systems, Vol. 3, pp. 201-217, 2007.

4. Krishnamurthy, T., and Gallegos, Adam M., "Damage Characterization Using the Extended Finite Element Method for Structural Health Management," AIAA-2011-1701, Presented at the 52nd AIAA/ASME/ASCE/ AHS/ASC Structures, Structural Dynamics and Materials Conference 13th AIAA Non-Deterministic Approaches Conference, Denver, CO, April 4-7, 2011.

5. Tessler, A., and Spangler, J., "A Least-Squares Variational Method for Full-Field Reconstruction of Elastic Deformations in Shear Deformable Plates and Shells," Computer Methods in Applied Mechanics and Engineering, Vol. 194, pp. 327-335, 2005.

6. Quach, C., Vazquez, S., Tessler, A., Moore, J., Cooper, E., and Spangler, J., "Structural Anomaly Detection using Fiber Optic Sensors and Inverse Finite Element Method," AIAA Guidance, Navigation, and Control Conference and Exhibit, San Francisco, CA. AIAA Paper 2005-6357, 2005.

7. Anonymous, DOT, Design Optimization Tools, User's Manual, Version 5.0, Vanderplaats Research \& Development, Inc., Colorado Springs, CO, 80906.

8. MathWorks, http://www.mathworks.com/help/toolbox/gads/. 